\title{
REVISITING THE LEGAL REGULATION OF ESSENTIAL CONDITIONS OF THE STATE (MUNICIPAL) CONTRACT IN THE LEGISLATION OF THE RUSSIAN FEDERATION
}

\author{
Olga A. Sergacheva \\ Volgograd Institute of Management - the Branch of the Russian Presidential Academy \\ of National Economy and Public Administration under the President of the Russian Federation, \\ Volgograd, Russian Federation
}

Introduction: in recent years, the study of the legal regulation of public procurement in general, and the state (municipal) contract in particular, is becoming increasingly important. This is due, primarily, to the difficulties of the law enforcement arising from the imperfect legal and technical work of the legislator when creating Federal law № 44-FZ of 05.04.2013 "On the Contract System of the Federal and Municipal Procurement of Goods, Works and Services". The issue of the essential conditions of the state (municipal) contract is debatable in the absence of such a term in the law, evidenced by the numerous civil law studies on the essential (mandatory, necessary) conditions of the state (municipal) contract as a kind of the civil contract. The purpose of the study is to reveal the main approaches to the understanding of the essential conditions of a civil contract and, in particular, the state (municipal) contract. Methods: there have been applied in aggregate the methods of scientific knowledge, including the basic methods of systemacity and analysis. Results: it is proved that the essential conditions of a civil contract are the conditions on the subject of the contract, the conditions which are named in the law or other legal acts as essential or necessary for the contracts of this type, and also all those conditions concerning which there must be reached an agreement upon the application of one of the parties. The requirements of Clause 1 of Article 432 of the RF Civil Code apply not only to civil law contracts, but also to state (municipal) contracts. The essential conditions of state (municipal) contracts should include both those regulated by Federal law № 44-FZ and those specified as essential for the certain types of state (municipal) contracts. Conclusions: the terminological inaccuracies do not affect the essence of the phenomenon, namely, with due regard to the reference by Article 432 of the Civil Code that the essential conditions are those which are named in the law or other legal acts as essential or necessary for the contracts of this type, the mandatory conditions, referred to in Federal law № 44-FZ, can be considered the essential conditions of the state (municipal) contract.

Keywords: state (municipal) contract, essential conditions, mandatory conditions, procurement for the state and municipal needs, civil contract.

УДК 346.34

ББК 65.272 .21

\section{К ВОПРОСУ О ПРАВОВОМ РЕГУЛИРОВАНИИ СУЩЕСТВЕННЫХ УСЛОВИЙ ГОСУДАРСТВЕННОГО (МУНИЦИПАЛЬНОГО) КОНТРАКТА В ЗАКОНОДАТЕЛЬСТВЕ РОССИЙСКОЙ ФЕДЕРАЦИИ}

\section{Ольга Александровна Сергачева}

Волгоградский институт управления - филиал Российской академии народного хозяйства и государственной службы при Президенте РФ, г. Волгоград, Российская Федерация

Введение: в последние годы исследование правового регулирования сферы публичных закупок в целом и государственного (муниципального) контракта в частности становится все более актуальным. Это 
обусловлено в первую очередь сложностями правоприменения, вытекающими из несовершенной юридико-технической работы законодателя при создании Федерального закона от 5 апреля 2013 г. № 44-Ф3 «О контрактной системе в сфере закупок товаров, работ, услуг для обеспечения государственных и муниципальных нужд». Вопрос о существенных условиях государственного (муниципального) контракта является дискуссионным в силу отсутствия такого термина в указанном законе, об этом свидетельствуют многочисленные цивилистические исследования, посвященные существенным (обязательным, необходимым) условиям государственного (муниципального) контракта как разновидности гражданско-правового договора. Цель исследования - раскрыть основные подходы к пониманию существенных условий гражданско-правового договора и, в частности, государственного (муниципального) контракта. Методы: применены в совокупности методы научного познания, среди которых основные - методы системности и анализа. Результаты: обосновано, что существенными условиями гражданско-правового договора являются условия о предмете договоpa, условия, которые названы в законе или иных правовых актах как существенные или необходимые для договоров данного вида, а также все те условия, относительно которых по заявлению одной из сторон должно быть достигнуто соглашение. При этом требования п. 1 ст. 432 ГК РФ распространяются не только на гражданско-правовые договоры, но и на государственные (муниципальные) контракты. К существенным условиям государственных (муниципальных) контрактов следует отнести как указанные в Федеральном законе № 44Ф3, так и указанные в качестве существенных для отдельных видов государственных (муниципальных) контрактов. Выводы: терминологические неточности не влияют на суть явления, а именно с учетом указаний статьи 432 Гражданского кодекса на то, что существенными являются условия, которые названы в законе или иных правовых актах как существенные или необходимые для договоров данного вида, обязательные условия, о которых речь идет в Федеральном законе № 44-Ф3, могут считаться существенными условиями государственного (муниципального) контракта.

Ключевые слова: государственный (муниципальный) контракт, существенные условия, обязательные условия, закупки для государственных и муниципальных нужд, гражданско-правовой договор.

UDC 346.34

BBK 65.272.21

\title{
LA QUESTIONE DELLA REGOLAMENTAZIONE GIURIDICA DEGLI ELEMENTI ESSENZIALI DI UN CONTRATTO PUBBLICO NELLA LEGISLAZIONE DELLA FEDERAZIONE RUSSA
}

\author{
Olga A. Sergacheva \\ Volgograd Institute of Management - un ramo dell'Accademia Presidenziale Russa dell'Economia Nazionale \\ e della Pubblica Amministrazione, Volgograd, Federazione Russa
}

Introduzione: negli ultimi anni lo studio della regolamentazione giuridica della sfera degli appalti pubblici in generale e degli contratti pubblici, in particolare, sta diventando sempre più importante. Ciò è dovuto, in primo luogo, le difficoltà di applicazione derivanti dal imperfetto lavoro di legislatore per creare la Legge federale del 04.05.2013 No. 44-FZ “A proposito di sistema contrattuale nel settore d'acquisto di beni, lavori e servizi per statali e municipali esigenze" (di seguito denominato - Legge federale No. 44-FZ) - si tratta degli appalti pubblici. La questione degli elementi essenziali di contratto pubblico è discutibile a causa della mancanza di un tale concetto nel suddetto Legge federale, evidenziato da numerosi studi scientifici sulle condizioni essenziali di contratto pubblico come una specie di contratto di diritto civile. Lo scopo della ricerca: rivelare i principali approcci alla comprensione degli elementi essenziali di un contratto di diritto civile, e in particolare, di un contratto pubblico. Metodi: è applicato un insieme dei metodi della conoscenza scientifica, tra cui i principali sono metodo di analisi e metodo sistematico. Risultati: è dimostrato che gli elementi essenziali di un contratto di diritto civile sono gli elementi che individuano l'oggetto del contratto, gli elementi che sono chiamati in una legge o negli altri atti giuridici come essenziali o necessarie per i contratti di questo tipo, ed anche tutti gli elementi per le quali, su richiesta di una delle parti, dovrebbe essere raggiunto un'accordo. I requisiti di cui par. 1, art. 432 del Codice Civile della Federazione Russa, si applicano non solo ai contratti di diritto civile, ma anche per i contratti pubblici. Ai sensi della Legge federale No. 44-FZ agli elementi essenziali dei contratti pubblici devono essere attribuiti quegli elementi che sanciti alla Legge, ed anche quegli che sono indicati come essenziali per alcuni tipi di contratti pubblici. Conclusioni: imprecisioni terminologiche non influenzano l'essenza del fenomeno, cioè, tenendo conto delle indicazioni del l'articolo 432 del 
Codice Civile della Federazione Russa in cui stabilisce che sono gli elementi essenziali quegli che sono chiamati in una legge o negli altri atti giuridici come essenziali o necessarie per i contratti di questo tipo, possiamo concludere che gli elementi obbligatori del contratto pubblico, di cui alla Legge federale No. 44-FZ, sono gli elementi essenziali di contratto pubblico.

Le parole chiave: il contratto pubblico, gli elementi essenziali, gli elementi obbligatori, gli appalti pubblici, il contratto di diritto.

\section{Introduzione}

Negli ultimi anni lo studio della regolamentazione giuridica della sfera degli appalti pubblici in generale e degli contratti pubblici, in particolare, sta diventando sempre più importante. La questione degli elementi essenziali di contratto pubblico è discutibile a causa della mancanza di un tale concetto nel suddetto Legge federale, evidenziato da numerosi studi scientifici sulle condizioni essenziali di contratto pubblico come una specie di contratto di diritto civile.

Le disposizioni in materia di appalti pubblici sono regolati in Russia i due principali atti giuridici normativi: il Codice Civile della Federazione Russa [5; 6] (di seguito denominato-CC FR) e della Legge federale del 05.04.2013 No. 44-FZ “A proposito di sistema contrattuale nel settore d'acquisto di beni, lavori e servizi per statali e municipali esigenze" [13] (di seguito denominato - Legge federale No. 44-FZ). La definizione generale di un contratto pubblico contiene parte 8 dell'articolo 3 della Legge federale No. 44-FZ: "contratto statale, il contratto comunaleun contratto concluso a nome della Federazione Russa, del soggetto della Federazione Russa (contratto statale) o della comune (contratto comunale) da una amministrazione pubblica per fornire rispettivamente, le esigenze dello stato, esigenze comunali”. Da questa definizione si deduce che il contratto pubblico è una specie di contratto di diritto civile, che ha, però, alcune specificità. Come giustamente sottolineato in letteratura, "uno dei concetti basilari del diritto civile è un contratto" [11].

Nel questo saggio vorremmo rivelare i principali approcci alla comprensione degli elementi essenziali di un contratto di diritto civile, e in particolare, di un contratto pubblico.

\section{Regolamentazione giuridica degli elementi essenziali di un contratto pubblico}

Le norme del CC FR (art. 420), in primo luogo, contengono una definizione generale del contratto: contratto è un accordo tra due o più persone sulla creazione, la modifica o la cessazione dei diritti ed obblighi civili. In secondo luogo, il CC FR dedica diversi articoli ai tipi diversi degli contratti pubblici. Essi comprendono, ad esempio, l'articoli 525-534 di paragrafo 4 del capitolo 30 del CC FR (il contratto pubblico per la fornitura di beni per 1'esigenze statali o municipali), l'articoli 763-768 di paragrafo 5 del capitolo 37 del CC FR (il contratto pubblico di lavori per l'esigenze statali o municipali), nonché le disposizioni generali del contratto per i servizi a pagamento che si applicano ai contratti pubblici di servizi per l'esigenze statali o municipali. Locazione di beni immobili può anche essere oggetto di contratto pubblico (questo si desume dalle disposizioni della parte 1 dell'art. 1 del paragrafo 3 della Legge federale No. 44-FZ), quindi, per tale contratto si applicano le disposizioni generali in materia di proprietà in affitto (in particolare, il paragrafo 1 e il paragrafo 4 capitolo 34 del CC FR).

Alcune caratteristiche salienti di un contratto pubblico si estende dettaglio nel suo studio L.V. Andreeva [1]. Tra questi, l'autore mette in evidenza: 1) una composizione soggettiva speciale; 2) lo scopo specifico del contratto; 3) l'uso di mezzi speciali per gli appalti pubblici; 4) il finanziamento della fornitura di beni a scapito dei fondi di bilancio, e ha attirato per questo scopo fonti extra-bilancio; 5) le norme speciali per l'esecuzione, la modifica e la cessazione degli contratti pubblici; 6) la complessità delle relazioni in evoluzione tra amministrazione pubblica e la seconda parte del contratto.

Perché il contratto pubblico, come detto sopra, è una specie di contratto di diritto civile, lo deve contenere alcune elementi essenziali (senza quali è impensabile alcun contratto civile). Nel CC FR secondo gli elementi essenziali si intendono gli elementi che individuano l'oggetto del contratto, gli elementi che sono chiamati in una legge o negli altri atti giuridici come essenziali o necessarie per i contratti di questo tipo, ed anche tutti gli elementi per le quali, su richiesta di una delle parti, dovrebbe 
essere raggiunto un'accordo (parte 1 dell'articolo 432 del CC FR). Tuttavia, nella scienza di diritto civile non esiste un approccio unico alla domanda, che tipo degli elementi del contratto dovrebbe essere classificato come essenziale. È interessante notare che anche nella dottrina estera civilistica non esiste ancora un unico punto di vista sulla questione della regolamentazione giuridica degli elementi essenziali dei contratti di diritto civile e dei contratti pubblici [10].

Come è già notato, nella scienza russa di diritto civile, ci sono diversi approcci alla definizione degli elementi essenziali del contratto. Così, ad esempio, V.V. Vitryansky scrive che gli elementi essenziali del contratto sono i seguenti: 1) gli elemento che individuano l'oggetto del contratto; 2) gli elementi necessari per questo tipo di contratto; 3) gli elementi che sono chiamati in una legge o negli altri atti giuridici come essenziali o necessarie per i contratti di questo tipo; 4) tutti gli elementi per le quali, su richiesta di una delle parti, dovrebbe essere raggiunto un' accordo [4]. La stessa opinione ha il professore E.A. Sukhanov [9]. Tuttavia, ci sono altri punti di vista sull'elenco degli elementi essenziali del contratto, in particolare, N.I. Solovyanenko indica che in relazione agli elementi essenziali del contratto "è tradizionalmente ritenuto necessario a raggiungere un accordo sui tre elementi seguenti: l'oggetto, il prezzo e la durata del contratto" [8], e N.V. Kagalnitskova individua tre gruppi degli elementi essenziali del contratto [7].

Come giustamente notato da F.A. Tasalov, "ovviamente che i requisiti parte 1, art. 432 del CC FR, si applicano non solo ai contratti di diritto civile, ma anche ai contratti pubblici" [12]. Considerate le specifiche di pubbliche relazioni nel settore degli appalti pubblici, nonché le disposizioni del parte 13 dell'articolo 34 della Legge federale No. 44-FZ, gli elementi essenziali dei contratti pubblici dovrebbe includere i seguenti: 1) i'oggetto di contratto pubblico; 2) il prezzo di contratto pubblico; 3) la condizione di un prezzo fisso di contratto pubblico; 4) l'ordine e la tempistica di accettazione dell'amministrazione pubblica della merce consegnata, lavoro svolto (i risultati) o servizi offerti in termini di conformità con la quantità, la completezza, requisiti di volume stabiliti dal contratto, nonché le procedure e ritardi nell'ottenimento di tali risultati di accettazione; 5) il periodo di esecuzione degli obblighi delle parti di contratto pubblico; 6) la responsabilità delle parti del contratto pubblico per inadempimento o la cattiva esecuzione di obblighi contrattuali. Va notato che nel testo della Legge federale No. 44-FZ questi elementi sono indicati non come essenziali, ma come obbligatori. Nella dottrina giustamente osservato che il concetto degli elementi essenziali non sono previsti nella legge, "l'elenco degli elementi contrattuali che possiamo chiamare essenziali non c'è" [2].

Siamo convinti che imprecisioni terminologiche non influenzano l'essenza del fenomeno, cioè, tenendo conto delle indicazioni del l'articolo 432 del Codice Civile della Federazione Russa in cui stabilisce che sono gli elementi essenziali quegli che sono chiamati in una legge o negli altri atti giuridici come essenziali o necessarie per i contratti di questo tipo, possiamo concludere che gli elementi obbligatori del contratto pubblico, di cui alla Legge federale No. 44-FZ, sono gli elementi essenziali di contratto pubblico.

Ulteriore attenzione dovrebbe essere prestata agli elementi essenziali delle singole tipologie dei contratti pubblici.

1. L'essenziali (obbligatori) elementi di contratto pubblico per la fornitura di beni per l'esigenze statali e municipali esigenze sono seguenti:

1) requisiti di qualità, tecnica e caratteristiche funzionali dei beni (comma 1 parte 1 dell'articolo 33 della Legge federale No. 44-FZ);

2) l'immagine di prodotto, che permette di identificarlo (se la merce fornita deve corrispondere all'immagine) (comma 4 parte dell'articolo 33 della Legge federale No. 44-FZ);

3 ) requisiti dei beni (nuovi o di seconda mano, riparato) (comma 7 parte 1 dell'articolo 33 della Legge federale No. 44-FZ);

4) l'indicazione dei Denominazione Comune Internazionale di farmaci, e in loro assenza - nomi di chimico raggruppamento, se il contratto è per la fornitura di medicinali (comma 6 della parte 1, l'articolo 33 della Legge federale No. 44-FZ);

5) i requisiti per il periodo di garanzia dei beni e (termini di garanzia della qualità o), per il costo delle merci è concesso per l'attuazione obbligatoria della sua installazione e messa in servizio, alla formazione delle persone impegnate nell'uso e manutenzione dei beni - se necessario, in caso di 
fornitura di macchine e attrezzature - se è previsto sulla documentazione del prodotto (parte 4 dell'articolo 33 della Legge federale No. 44-FZ);

6) l'obbligo di fornire garanzie del produttore dei beni e (o) il fornitore e la sua validità nel caso della fornitura di nuove macchine e attrezzature (parte 4 dell'articolo 33 della Legge federale No. 44-FZ);

7) informazioni sul numero, luogo di consegna e la durata della consegna della merce (comma 2, art. 42 della Legge federale No. 44-FZ).

E' anche importante considerare gli elementi contrattuali che si applicano a un contratto civile di fornitura dei beni.

2. L'essenziali (obbligatori) elementi di contratto pubblico per l'esecuzione di lavori per l'esigenze statali e municipali:

1) i requisiti per la qualità, le caratteristiche tecniche e funzionali dei lavori (comma 1, parte 1, 1'articolo 33 della Legge federale No. 44-FZ);

2) i requisiti per il periodo di garanzia del lavoro e (o) il volume di garanzia della qualità (parte 4 dell'articolo 33 della Legge federale No. 44-FZ);

3) posto di lavoro (parte 2 dell'articolo 42 della Legge federale No. 44-FZ);

4) il periodo di completamento del lavoro (parte 2 dell'articolo 42 della Legge federale No. 44-FZ);

5) quantità di lavoro da eseguire (parte 1, l'articolo 766 del CC FR);

6) i costi del lavoro da eseguire (parte 1, l'articolo 766 del CC FR);

7) date di inizio e fine dei lavori (parte 1, l'articolo 766 del CC FR);

8) la quantità e la procedura di finanziamento e di pagamento per il lavoro (parte 1, 1'articolo 766 del CC FR);

9) i modi per garantire l'adempimento degli obblighi delle parti (parte 1, l'articolo 766 del CC FR).

3. L'essenziali (obbligatori) elementi di contratto pubblico per la fornitura di servizi per l'esigenze statali e municipali:

1) i requisiti per la qualità, le caratteristiche tecniche e funzionali dei servizi (comma 1, parte 1 dell'articolo 33 della Legge federale No. 44-FZ);

2) i requisiti per il servizio di garanzia e (o) il volume di garanzia della qualità (parte 4 dell'articolo 33 della Legge federale No. 44-FZ);

3) il luogo delle prestazioni di servizi (parte 2 dell'articolo 42 della Legge federale No. 44-FZ);
4) la programma di prestazioni di servizi (parte 2 dell'articolo 42 della Legge federale No. 44-FZ).

Va notato che parte 1 dell'articolo 95 della Legge federale No. 44-FZ stabilisce un elenco limitato di casi in cui gli elementi essenziali del contratto possono essere modificate. Questi casi includono, per esempio, una diminuzione di fondi di bilancio limiti precedentemente comunicati all'amministrazione pubblica come il destinatario degli obblighi di bilancio. In questo caso l'amministrazione pubblica nel corso di esecuzione del contratto prevede il coordinamento delle nuove condizioni contrattuali, compreso il prezzo e (o) i termini del contratto $\mathrm{e}(\mathrm{o})$ la quantità delle merci, il volume di lavori o servizi che sono un'oggetto del contratto, in conformità con parte 6 dell'art. 161 del Codice di bilancio della Federazione Russa [3].

Oltre a ridurre i fondi di bilancio gli elementi essenziali del contratto possono essere modificate anche nel caso della riduzione del prezzo del contratto senza modificare il volume e la qualità del lavoro svolto, o aumentare o diminuire la quantità di lavoro per non più del $10 \%$, con un corrispondente aumento o una diminuzione del prezzo del contratto. La possibilità di tali cambiamenti nelle condizioni deve essere prevista nella documentazione degli appalti pubblici e nel contratto pubblico.

Inoltre, in alcuni casi, i condizioni del contratto possono essere cambiati senza clausola di possibilità di tali cambiamenti nella documentazione dell'appalto e nel contratto, ad esempio, se in conformità con le leggi cambiano i prezzi regolamentati dallo Stato (tariffe).

\section{Conclusioni}

Imprecisioni terminologiche non influenzano l'essenza del fenomeno, cioè, tenendo conto delle indicazioni del l'articolo 432 del Codice Civile della Federazione Russa in cui stabilisce che sono gli elementi essenziali quegli che sono chiamati in una legge o negli altri atti giuridici come essenziali o necessarie per i contratti di questo tipo, possiamo concludere che gli elementi obbligatori del contratto pubblico, di cui alla Legge federale No. 44-FZ, sono gli elementi essenziali di contratto pubblico.

Ulteriore attenzione dovrebbe essere prestata agli elementi essenziali delle singole 
tipologie dei contratti pubblici.

1. L'essenziali (obbligatori) elementi di contratto pubblico per la fornitura di beni per l'esigenze statali e municipali esigenze.

2. L'essenziali (obbligatori) elementi di contratto pubblico per l'esecuzione di lavori per l'esigenze statali e municipali.

3. L'essenziali (obbligatori) elementi di contratto pubblico per la fornitura di servizi per per l'esigenze statali e municipali.

Speriamo che l'analisi degli elementi essenziali di contratti pubblici di cui abbiamo trascorso in questo articolo, permette a prevenire i problemi verificati a causa di imprecisione e mancanza di formulazione di concetto degli elementi essenziali del contratto pubblico nella legislazione della Federazione Russa. E' molto importante superare le lacune legislative; si può affermare che è il compito più importante di moderno diritto civile russo.

\section{REFERENCES}

1. Abova T.E., Kabalkin A.Yu., eds. Kommentariy k Grazhdanskomu kodeksu Rossiyskoy Federatsii, chasti pervoy [Commentary on the Civil Code of the Russian Federation, Part One]. Moscow, Yurayt-Izdat Publ., 2007.

2. Andreeva L.V. Zakupki tovarov $i$ energoservisnykh rabot dlya federalnykh gosudarstvennykh nuzhd: pravovoe regulirovanie [Procurement of Goods and Energy Services for Federal State Needs: Legal Regulation]. Moscow, 2011.

3. Belyaeva O.A. Zakupka u edinstvennogo postavshchika: novatsii kontraktnoy sistemy [Purchase from a Single Supplier: Innovation of the Contract System]. Konkurentsiya i pravo, 2013, no. 4.

4. Byudzhetnyy kodeks Rossiyskoy Federatsii ot 31.07.1998 № 145-FZ [Budget Code of the Russian Federation of July 31, 1998 no. 145-FL]. Sobranie zakonodatelstva RF, 1998, August 3 (no. 31), art. 3823.

5. Federalnyy zakon «O kontraktnoy sisteme v sfere zakupok tovarov, rabot, uslug dlya obespecheniya gosudarstvennykh i munitsipalnykh nuzhd» ot 05.04.2013 № 44-FZ [Federal Law “On Contract System in the Sphere of Goods, Works and
Services Purchase for Meeting State and Municipal Needs"of April 5, 2013 no. 44-FL]. Sobranie zakonodatelstva RF, 2013, April 8 (no. 14), art. 1652.

6. Grazhdanskiy kodeks Rossiyskoy Federatsii (chast pervaya) ot 30.11.1994 № 51-FZ [Civil Code of the Russian Federation (Part One) of November 30, 1994 no. 51-FL]. Sobranie zakonodatelstva RF, 1994, December 5 (no. 32), art. 3301.

7. Grazhdanskiy kodeks Rossiyskoy Federatsii (chast vtoraya) ot 26.01.1996 № 14-FZ [Civil Code of the Russian Federation (Part Two) of January 26, 1996 no. 14-FL]. Sobranie zakonodatelstva RF, 1996, January 29 (no. 5), art. 410.

8. Kagalnitskova N.V. Nekotorye aspekty nezaklyuchennogo i nedeystvitelnogo dogovora $\mathrm{V}$ usloviyakh reformirovaniya grazhdanskogo zakonodatelstva [Some Aspects of Non-Concluded and Invalid Contract in the Conditions of Civil Legislation Reformation]. Vestnik Volgogradskogo gosudarstvennogo universiteta. Seriya 5, Yurisprudentsiya [Science Journal of Volgograd State University. Jurisprudence], 2015, no. 4 (29).

9. Rossiyskoe grazhdanskoe pravo: T. II: Obyazatelstvennoe pravo [Russian Civil Law. Vol. 2: Obligation Law]. Moscow, Statut Publ., 2011.

10. Sergacheva O.A. K voprosu o pravovoy prirode publichnogo kontrakta $v$ sootvetstvii s zakonodatelstvom Italyanskoy Respubliki [On the Juridical Nature of Public Contract in Accordance with Legislation of Italian Republic]. Grazhdanskoe pravo, 2014, no. 1.

11. Simaeva E.P. Sistema dogovornogo (kontraktnogo) prava v Rossii i Kanade: sravnitelnopravovoy analiz [The System of Contract Law in Russia and Canada: Comparative Legal Analysis]. Vestnik Volgogradskogo gosudarstvennogo universiteta. Seriya 5 , Yurisprudentsiya [Science Journal of Volgograd State University. Jurisprudence], 2011, no. 1 (14).

12. Tasalov F.A. Kontraktnaya sistema v sfere gosudarstvennykh zakupok Rossii i SShA: sravnitelno-pravovoe issledovanie [Contract System in the Sphere of State Purchases of Russia and the USA: Comparative Legal Research]. Moscow, Prospekt Publ., 2014.

13. Vitryanskiy V.V. Nekotorye itogi kodifikatsii pravovykh norm o grazhdansko-pravovom dogovore [Some Results of Codification of Legal Regulations on Civil-Legal Contract]. Medvedev D.A., ed. Kodifikatsiya rossiyskogo chastnogo prava [Codification of the Russian Private Law]. Moscow, Statut Publ., 2008. 


\section{ВОПРОСЫ ЧАСТНОПРАВОВОГО РЕГУЛИРОВАНИЯ}

\section{Information About the Author}

Olga A. Sergacheva, Candidate of Juridical Sciences, Assistant Professor, the Department of Civil Law Disciplines, Volgograd Institute of Management - the Branch of the Russian Presidential Academy of National Economy and Public Administration under the President of the Russian Federation, Gagarina St., 8, 400131 Volgograd, Russian Federation, sergacheva.olia@yandex.ru.

\section{Информация об авторе}

Ольга Александровна Сергачева, кандидат юридических наук, доцент кафедры гражданско-правовых дисциплин, Волгоградский институт управления - филиал Российской академии народного хозяйства и государственной службы при Президенте РФ, ул. Гагарина, 8, 400131 г. Волгоград, Российская Федерация, sergacheva.olia@yandex.ru.

\section{Informazioni sull'autore}

Olga A. Sergacheva, Candidato di scienza giuridica, docente presso, la Facoltà di Diritto, Volgograd Institute of Management - un ramo dell'Accademia Presidenziale Russa dell'Economia Nazionale e della Pubblica Amministrazione, via Gagarin, 8, 400131 Volgograd, Federazione Russa, sergacheva.olia@yandex.ru. 